\title{
Elderly with Autism: Executive Functions and Memory
}

\author{
Hilde M. Geurts • Marlies E. Vissers
}

Published online: 8 June 2011

(c) The Author(s) 2011. This article is published with open access at Springerlink.com

\begin{abstract}
Cognitive autism research is mainly focusing on children and young adults even though we know that autism is a life-long disorder and that healthy aging already has a strong impact on cognitive functioning. We compared the neuropsychological profile of 23 individuals with autism and 23 healthy controls (age range 51-83 years). Deficits were observed in attention, working memory, and fluency. Aging had a smaller impact on fluency in the high functioning autism (HFA) group than in the control group, while aging had a more profound effect on visual memory performance in the HFA group. Hence, we provide novel evidence that elderly with HFA have subtle neuropsychological deficits and that the developmental trajectories differ between elderly with and without HFA in particular cognitive domains.
\end{abstract}

Keywords Autism $\cdot$ ASD $\cdot$ Aging $\cdot$ Cognition ·

Neuropsychology

\section{Introduction}

Cognitive changes are common when people age, but what happens with aging if one has a psychiatric disorder like autism, that is, what if one already has cognitive deficits? Will everything deteriorate faster, or do efficient

H. M. Geurts $(\bowtie)$

Brain and Cognition, Department of Psychology,

University of Amsterdam, Roetersstraat 15,

1018 WB Amsterdam, The Netherlands

e-mail: h.m.geurts@uva.nl

M. E. Vissers

Cognitive Science Center Amsterdam, Amsterdam,

The Netherlands compensatory mechanisms develop? At present, it is unknown what happens when people with autism age (Rapin and Tuchman 2008; Seltzer et al. 2004; Volkmar et al. 2004).

Autism $^{1}$ is a heterogeneous psychiatric disorder which is characterized by social and communicative impairments and restricted, stereotypical patterns of behavior and interests (American Psychiatric Association (APA) 1994, 2000; Kanner 1943; Volkmar et al. 2004). The impairments are thought to last a lifetime (Cederlund et al. 2008; Howlin et al. 2004). Even though some autism symptoms seem to decrease with increasing age, elderly with autism will not reach normal levels of social functioning. A handful of studies focused on elderly with autism (James et al. 2006; Raznahan et al. 2010; Seltzer et al. 2004), but studies focusing on cognition in autism are mainly restricted to childhood and early adulthood. From these studies with young individuals it seems that autism is associated with early neurodevelopmental deficits in the same cognitive mechanisms that are also known to deteriorate with aging.

In both childhood and adulthood individuals with autism show a broad range of cognitive deficits, including executive functioning (EF) deficits (Geurts et al. 2009; Hill 2004; Pennington and Ozonoff 1996; Sergeant et al. 2002). $\mathrm{EF}$ is an umbrella term to describe various problems in complex, goal directed actions, and encompasses different cognitive domains such as working memory, cognitive flexibility, planning, and fluency. The idea has been postulated that the developmental pattern of EF in children and

\footnotetext{
1 The term autism is used to refer to autism spectrum disorders including autism, Asperger syndrome, and Pervasive Developmental Disorder-Not Otherwise Specified.
} 
adolescents with autism is atypical (Happe et al. 2006; Luna et al. 2007; Pellicano 2010).

This idea stems from the finding that children with autism between 8 and 11 years of age showed several EF deficits, while these deficits did not emerge in children with autism aged 11-16 years (Happe et al. 2006). Also, in a recent longitudinal 3 year follow up, planning capacity in children with autism improved at a faster rate than that of typically developing children (Pellicano 2010). Hence, these findings indicate that EF deficits abate when aging. However, these findings might be in contrast with most recent adult studies in which executive dysfunctions are still present in individuals with autism above 16 years of age (Ambery et al. 2006; Bramham et al. 2009; Goldstein et al. 2001; Hill and Bird 2006; Keary et al. 2009; Lopez et al. 2005; Minshew et al. 2002; Shafritz et al. 2008). In some of these studies even elderly were included (Ambery et al. 2006; Hill and Bird 2006), but while the age range of participants was often large (16-66 year), the effect of age was not explored. In a cross-sectional developmental study (Luna et al. 2007) executive dysfunctions were present in people with autism of different ages $(8-12,13-17$, and 18-33 year). Across the three age groups the autism group encountered inhibitory control deficits as well as working memory deficits. However, developmental improvements in inhibitory control were similar in both groups (i.e., parallel development), while the development of working memory was impaired in the autism group (Luna et al. 2007). The smaller extent of improvements in EF in children has also been reported in two longitudinal studies (Griffith et al. 1999; Ozonoff and McEvoy 1994). The combined findings from cross sectional and longitudinal studies suggest that there are different developmental patterns for different aspects of EF. Whether elderly with autism still encounter executive dysfunctions depends on whether deficits associated with autism at young age will remain stable, become more severe, or abate.

Unfortunately, none of the few autism-aging studies (James et al. 2006; Raznahan et al. 2010; Seltzer et al. 2004) systematically examined EF in elderly with autism. However, in case-studies of five elderly people with autism it has been described that each case still encountered $\mathrm{EF}$ deficits (James et al. 2006). Since EF-profiles in children and adults with autism show similarities to those seen in healthy elderly, it is surprising that EF has not yet been thoroughly studied in aging individuals with autism. A large body of research on aging in typically developing adults revealed that aging is associated with declines in various cognitive domains (Goh and Park 2009; Salthouse 2004). However, age-related deterioration in EF is thought to underlie most of these age-related declines (Friedman et al. 2009; Salthouse and Meinz 1995; Salthouse and
Miles 2002; Verhaeghen and Cerella 2002), such as the decline in memory performance (Friedman et al. 2009; Salthouse 2004). It seems that memory itself is not impaired in adults with autism (Bowler et al. 2009; Bramham et al. 2009), but it is unknown whether memory deficits are present in elderly with autism.

The cognitive challenges healthy elderly people encounter are thought to relate to the structural (Galuzzi et al. 2008; Gunning-Dixon et al. 2009; Mora et al. 2007; Sullivan and Pfefferbaum 2006) and functional changes (Cabeza 2002; Davis et al. 2008; Goh and Park 2009; Park and Reuter-Lorenz 2009) of the brain. Aging is especially thought to affect various frontal brain regions (Galluzzi et al. 2008; MacPherson et al. 2002), which have also been found to function atypically in individuals with autism when performing EF tasks (Gilbert et al. 2008; Just et al. 2007; Kana et al. 2007; Luna et al. 2002, 2007; Schmitz et al. 2006). How the combination of aging and autism affects brain anatomy and brain functioning, and thereby behavior, is largely unknown. The current study will, therefore, provide a direct comparison between elderly with high functioning autism (HFA) and age and gender matched controls on various neuropsychological tests to determine which cognitive deficits are present in elderly with HFA. In this way we can explore whether the deficits are still present or indeed abate as has been suggested in developmental studies focusing on children (e.g. Happe et al. 2006; Pellicano 2010).

\section{Methods}

Participants

\section{Autism Group}

Participants with autism were recruited via an advertisement in the magazine of the Dutch society for Autism and were included when they received a clinical diagnosis of autism, when they had a high score on the Social Responsiveness Scale (SRS, Constantino and Todd 2005; Dutch version; De la Marche et al. 2009), or when they met both criteria. A score of 60 or higher on the SRS is indicative of the presence of autism (Constantino and Todd 2005). Twenty participants had an official diagnosis made by an autism expert team: 16 were diagnosed with Asperger syndrome, 2 with autism, 1 with PDD-NOS, and 1 with ASD. For the remaining three participants an expert had indicated the presence of ASD, but did not give an official diagnose due to the old age of the person. Each of these three participants had a score higher than 60 on the SRS and were, therefore, included. 


\section{Control Group}

Controls were recruited via personal networks of the experimenters and none of the 23 control participants suffered from a psychiatric disorder, except for one participant who had suffered from a depression in the past which had been treated successfully. Also the relatives of these participants did not suffer from autism or any other psychiatric disorders.

The individual matching on age and gender was successful as the groups did not differ from each other with respect to age, and gender. Moreover, the groups were also matched on estimated IQ (range 81-130) and educational level (see Table 1). Given the educational level and the estimated IQ all participants were cognitively high functioning. As expected, the participants of the autism group reported more autism characteristics on the SRS when compared to controls.

\section{Materials}

\section{Verbal Intelligence}

The Dutch Adult Reading Test (DART; Schmand et al. 1992; Dutch version of the National Adult reading Test; Nelson 1982) was administrated as an estimator of verbal intelligence (DART-IQ) as reading ability of irregular words is relatively insensitive to cognitive impairment caused by neurological disorders and is a strong predictor of intelligence (Schmand et al. 1998).

The participant was presented with a list of irregular words of increasing morphological complexity which were to be read aloud. The number of correctly pronounced words was used to calculate a DART-IQ score, which was the main dependent measure.

\section{Processing Speed}

To assess general motor processing speed the WAIS-III Digit Symbol-copy (Wechsler 2002) was administered. Processing speed was assessed since it is often reduced in healthy aging (Salthouse 2004; Salthouse and Meinz 1995), and in children and adults with autism (Geurts et al. 2008; Spek et al. 2008).

Within 2 min participants needed to copy simple symbols shown on top of the form in empty squares in a particular order indicated by numbers above the squares. The dependent measure was the number of correctly copied symbols.

\section{Attention}

The Sustained Attention to Response Test (SART; Robertson et al. 1997) was administrated as in healthy elderly no impairments were present on this task (Carriere et al. 2010), while children with autism were impaired on this task (Johnson et al. 2007).

During the SART, single digits between ' 0 ' and ' 9 ' appeared on the screen in a randomized order $(250 \mathrm{~ms})$. Each digit was followed by a response cue (a cross in the middle of the screen). Participants were instructed to respond to a digit as soon as the response cue appeared, except when the digit was a ' 3 '. It was emphasized that responding correctly was just as important as responding quickly. Responses were given with a button press with the index finger of the preferred hand. The total inter-stimulus interval lasted 1,000 ms (response cue plus black screen following response). The experiment started off with a practice block consisting of 18 trials and was followed by 6 test blocks of 36 trials. This resulted in a total task duration of approximately $10 \mathrm{~min}$.

Table 1 Group means and standard deviations for age, gender, estimated IQ, educational level, and autism rating scale score

\begin{tabular}{llll}
\hline & Groups & & Statistics \\
\cline { 2 - 4 } & Autism $(n=23)$ & Controls $(n=23)$ & $F<1, n s, \eta^{2}=.00$ \\
\hline Age & 63.6 years $($ SD 7.5$)$ & $63.7($ SD 8.1$)$ & $\chi^{2}(1, N=46)=.00, p=1.0$ \\
Gender & $5 \mathrm{~F}, 18 \mathrm{M}$ & $5 \mathrm{~F}, 18 \mathrm{M}$ & $F<1, n s, \eta^{2}=.00$ \\
DART-IQ & $109.5(10.3)$ & $109.8(7.9)$ & $\chi^{2}(3, N=46)=1.679, p=.64$ \\
Education $^{a}$ & $1 / 6 / 7 / 9$ & $0 / 5 / 10 / 8$ & $F(1,44)=129.9, p<.001, \eta^{2}=.75$ \\
SRS $^{b}$ & $94.1(26.4)$ & $25.9(11.3)$ &
\end{tabular}

$F$ female, $M$ male, DART-IQ Dutch adult reading Test IQ, SRS Social Responsiveness Scale. Please note that various participants (autism group $n=12$; controls $n=14$ ) used medication to treat a broad range of conditions (e.g., diabetes, arthritis). None of these drugs affect performance on the tasks included in the current study

${ }^{a}$ The numbers between the slashes indicate the number of participants who had pre-vocational education/junior general secondary or vocation education/senior general secondary education or vocation colleges/university education

b The adult version of the SRS is a 65 -item questionnaire which addresses reciprocal social behavior, communicative deficits, and stereotypic behavior. The SRS is effective in distinguishing between individuals with and without autism. SRS scores range from 0 (socially highly competent) to 195 (severely socially impaired) 
Trials were analyzed per test block. The dependent measures were the mean reaction time for correct responses (MRT), the standard deviation of the MRT, the number of commission errors (incorrectly pressing the response key) and the number of omission errors (not pressing the response key when a response was required).

\section{Working Memory}

The subtest Spatial Span (forward problems) from the Wechsler Memory Scale-III (WMS; Wechsler 1987, 2002) was administrated to tap visuo-spatial working memory. Visuo-spatial working memory is impaired in healthy aging (Wilde et al. 2004) and is impaired in children and adults with autism (Geurts et al. 2004; Zinke et al. 2010).

The examiner tapped a sequence of squares, which the participant needed to repeat subsequently in the correct sequential order. The capacity of visuo-spatial working memory was measured by increasing the length of the modeled sequences. A sequence of two squares was the lowest memory load and a sequence of eight the highest memory load. The test ended when participants failed to complete at least one of the two trials of a particular difficulty level or when all 16 trials were administrated. The sum of the number of correctly reproduced trials in each of the difficulty levels was the dependent measure.

\section{Cognitive Flexibility}

The Modified Card Sorting Test (MCST; Nelson 1976) and the Trail Making Test (TMT; Reitan and Wolfson 1985) were administrated to measure cognitive flexibility.

Aging has a negative impact on performance on the MCST (Cafarra et al. 2004), which is an adapted version of the Wisconsin Card Sorting test (WCST). Both are sorting tasks that require participants to determine how to sort cards according to unknown categories. The participants needed to infer the sorting rule based on feedback given by the experimenter. After a fixed number of consecutive correct trials, the sorting rule changed. Hence, the participant needed to suppress the previous sorting rule and discover the new sorting rule. In contrast to the WCST, in the MCST a warning that the sorting rule needs to be changed is included. The dependent measure of interest was the number of perseverative errors. On the WCST children and adults with autism generally fail (Geurts et al. 2009), but this task is not suitable for elderly. This is why the MCST was chosen even though adults with autism do not seem to fail on this task (Barnard et al. 2008; Hill and Bird 2006).

The TMT is a timed task that requires the participant to connect a series of letters and numbers in ascending order. In the first part (A) only numbers need to be connected in the right order (from 1 to 2 , from 2 to 3 , and so on till 25) and no letters are presented. In the second part (B) the participants need to alternate between numbers and letters (from 1 to A, from A to 2, from 2 to $\mathrm{B}$, and so on till 13). Dependent measure on this task was the difference between the time it took the participants to complete part A and part B (Reitan and Wolfson 1985). Aging has a negative impact on performance on the TMT (McCurry et al. 2001). Findings with respect to autism seem to suggest that children and adults with autism have difficulties with this task (Goldstein et al. 2001; Hill and Bird 2006; Minshew et al. 2002, but see Geurts et al. 2009).

\section{Planning}

The Tower of London of the Drexel University (ToL-DX; Culbertson and Zillmer 2001) was used to measure planning capacities. In aging studies it was found that elderly perform worse on the ToL than younger adults (Gilhooly et al. 1999; Zook et al. 2006). In autism research, the ToL has been found to be one of the most consistent indicators of planning deficits in children and adults with autism (Joseph et al. 2005; Pellicano et al. 2006; Robinson et al. 2009; Verte et al. 2005; Wallace et al. 2009; Zinke et al. 2010).

On the ToL-DX, participants were presented with a fixed start position of three colored beads placed on three pegs of different lengths on a wooden board. Participants were instructed to move beads from the start position to a predetermined goal position, while adhering to several rules. Ten different goal configurations were presented to the participants. The demand for planning increased as the task proceeded, as the minimum number of moves required to solve the problems increased from four per problem at the start of the task to seven at the end of the task. The dependent measure was the number of moves participants made on the task, of which the minimal number of moves required to solve the problems was subtracted.

\section{Fluency}

To measure verbal letter fluency, i.e. the capacity to generate novel responses, an adaptation of the Controlled Word Association Task (COWAT; Benton and Hamsher 1976) was administered. Performance on verbal fluency tasks decreases with increasing age (Clark et al. 2009). Also, children and adults with autism (Bramham et al. 2009; Geurts et al. 2004; Spek et al. 2008) generate less novel responses as compared to controls.

Participants were required to name as many words starting with a particular letter as they could within $60 \mathrm{~s}$. This was done for D, A, and S. Measure of interest was the sum of the number of correctly named words within $1 \mathrm{~min}$ for each letter. 


\section{Visual Memory}

Visual Reproduction of the WMS-III (Wechsler 1987, 2002) measures memory for visual stimuli. Administration of this task in healthy older adults shows age-associated impairments (Price et al. 2004). With respect to autism, no deficits seem to be present (Williams et al. 2006).

Participants looked at five geometrical figures for $10 \mathrm{~s}$. Immediately after viewing the figures and $20 \mathrm{~min}$ after viewing, participants were asked to reproduce the figures. Dependent measures were the degree to which the copied figure resembled the original figure (see Wechsler scoring manual; Wechsler 2002) on immediate and delayed reproduction.

\section{Verbal Memory}

The Dutch version of the Rey Auditory Verbal Learning Task (RAVLT; Rey 1964; Dutch version; Van den Burg et al. 1985) was administered to measure verbal memory. In aging research, the RAVLT is one of the most commonly used verbal learning tasks (Vakil et al. 2010). Adults with autism seem not to have difficulties with this specific task (Bowler et al. 2009).

Participants had to memorize a list of 15 unrelated words in five learning trials. Immediate recall was tested after each learning trial. Delayed recall and recognition were measured after a 20 min delay. In the recognition test the memorized words were intermixed with new words. The dependent measures were the total number of correctly recalled words at immediate and delayed recall, and the number of correctly recognized words.

\section{Procedure}

After participants had filled out an informed consent form and some questionnaires, they were tested. To exclude order effects, four fixed test orders were counterbalanced across participants. All participants received a small gift at the end of the test session, reports of the overall study findings, and a surprise gift voucher (15 euro). The study was approved by the local ethical committee. All procedures were conducted in accord with relevant laws and institutional guidelines.

\section{Results}

\section{Group Comparisons}

The alpha level was adjusted to compensate for the number of comparisons made. For each ANOVA or MANOVA alpha was set at .006 . As can be seen in Table 2, for most tasks no significant group effects were present. Nonetheless, the groups differed significantly on attention, working memory, and fluency. These findings will be discussed below.

\section{Attention}

For the SART a repeated measure ANOVA was conducted with group (2 levels) as between-subject factor and block (6 levels) as within-subject factor. There was a marginally significant interaction effect between group and block, $F(5$, $40)=3.07, p<.02, \eta^{2}=.28$ (see Fig. 1). The time course of the task (i.e., blocks) had a more pronounced effect in the control than in the autism group. While at the end of the six blocks both groups had similar MRTs, at the beginning of the task the controls responded slower than the autism group. Hence, controls started off slower than the autism group, but increased their speed when the task progressed, while the autism group hardly increased their speed over time. This observation was confirmed by additional independent $t$-tests, which indicated that elderly with autism were faster than controls in the second block, $t(44)=-2.17, p<.05$, but not in the last block (ns). While all participants were faster at the end of the task as compared to the beginning, $F(5,40)=3.14, p<.02$, $\eta^{2}=.28$, the interaction effect suggests that this was mainly due to the change in speed of responding in the control group. No significant differences emerged for the standard deviation of MRT (block: $F(5,40)=.91$, $n s$, $\eta^{2}=.19$; interaction block * group: $F<1, n s, \eta^{2}=.01$ ).

The controls made less commission errors than the autism group, but there was neither a significant effect of block, $F(5,40)=1.04, n s, \eta^{2}=.11$, nor a significant interaction effect between block and group, $F(5$, $40)=1.58, n s, \eta^{2}=.17$. Hence, accuracy did not improve or decrease across the task. For the number of omission errors no significant differences emerged (block: $F(5$, $40)=1.71, n s, \eta^{2}=.17$; interaction block $*$ group: $F(5$, 40) $=1.17, n s, \eta^{2}=.13$ ).

\section{Working Memory}

On the spatial span task the controls outperformed the autism group. The autism group remembered significantly less sequences than the controls. Thus, elderly with autism showed visual working memory problems.

\section{Fluency}

The total number of correct words was, although marginally, significantly higher in the controls than in the autism group. This implies that elderly with autism had difficulties with generating novel responses. 
Table 2 Group means, standard deviations, and statistics for each neuropsychological test

\begin{tabular}{|c|c|c|c|c|c|}
\hline \multirow[t]{2}{*}{ Domain } & \multirow[t]{2}{*}{ Task } & \multirow[t]{2}{*}{ Dependent measure } & \multicolumn{2}{|l|}{ Groups } & \multirow[t]{2}{*}{ Statistics } \\
\hline & & & Autism & Controls & \\
\hline Processing speed & Symbol copy & Wechsler score & $10.4(2.7)$ & $11.1(1.9)$ & $F(1,44)=1.16, n s, \eta^{2}=.03$ \\
\hline Attention & SART & $\begin{array}{l}\text { MRT } \\
\text { SD MRT } \\
\text { Commission errors } \\
\text { Omission errors }\end{array}$ & $\begin{array}{c}206.1(106.1) \\
98.0(63.0) \\
4.7(\mathbf{3 . 7}) \\
7.3(17.6)\end{array}$ & $\begin{aligned} & 251.4(99.3) \\
& 88.5(29.1) \\
& \mathbf{2 . 4}(\mathbf{2 . 2}) \\
& 0.8(1.7)\end{aligned}$ & $\begin{array}{l}F(1,44)=2.24, n s, \eta^{2}=.05 \\
F<1, n s, \eta^{2}=.01 \\
\boldsymbol{F}(\mathbf{1}, \mathbf{4 4})=\mathbf{6 . 2}, \boldsymbol{p}<. \mathbf{0 2}, \boldsymbol{\eta}^{\mathbf{2}}=\mathbf{. 1 2} \\
F(1,44)=3.12, n s, \eta^{2}=.07\end{array}$ \\
\hline Working memory & Spatial span & Total no correct & $6.6(1.7)$ & $8.1(1.7)$ & $F(1,44)=8.97, p<.005, \eta^{2}=.17$ \\
\hline Cognitive flexibility & $\begin{array}{l}\text { MCST } \\
\text { TMT }\end{array}$ & $\begin{array}{l}\text { No. of perseverations } \\
\text { Time B-Time A }(\mathrm{sec})\end{array}$ & $\begin{array}{c}3.3(5.6) \\
46.3(24.9)\end{array}$ & $\begin{array}{c}1.7(2.1) \\
36.5(16.8)\end{array}$ & $\begin{array}{l}F(1,44)=1.65, n s, \eta^{2}=.04 \\
F(1,44)=2.46, n s, \eta^{2}=.05\end{array}$ \\
\hline Planning & ToL-DX & Total moves & $27.1(13.1)$ & $30.8(17.6)$ & $F<1, n s, \eta^{2}=.02$ \\
\hline Fluency & COWAT & Total no correct & $40.2(9.8)$ & $46.7(9.2)$ & $F(1,44)=5.45, p<.03, \eta^{2}=.11$ \\
\hline Visual Memory & WMS figures & $\begin{array}{l}\text { Direct recall total score } \\
\text { Delayed recall total score }\end{array}$ & $\begin{array}{l}78.2(18.3) \\
57.6(25.0)\end{array}$ & $\begin{array}{l}82.5(9.1) \\
61.6(18.0)\end{array}$ & $\begin{array}{l}F(1,44)=1.05, n s, \eta^{2}=.02 \\
F<1, n s, \eta^{2}=.01\end{array}$ \\
\hline Verbal memory & RAVLT & $\begin{array}{l}\text { Direct recall total correct } \\
\text { Delayed recall total correct } \\
\text { Recognition total correct }\end{array}$ & $\begin{aligned} 45.4 & (10.0) \\
9.3 & (3.6) \\
29.2 & (1.3)\end{aligned}$ & $\begin{array}{r}45.1(8.3) \\
8.8(3.3) \\
28.2(2.2)\end{array}$ & $\begin{array}{l}F<1, n s, \eta^{2}=.00 \\
F<1, n s, \eta^{2}=.01 \\
F(1,44)=3.15, n s, \eta^{2}=.07\end{array}$ \\
\hline
\end{tabular}

In bold the measures on which significant differences emerged. For each group and for each dependent measure, participants with extreme scores were identified. Extreme scores were values more than three box plot lengths (i.e., standard errors) from the upper or lower edge of the box. Only for the SART and the MCST there were outliers in the autism and control group, but exclusion of these outliers did not alter the pattern of findings

WAIS Wechsler Adult Intelligence Scale, SART sustained attention to response test, WMS Wechsler Memory Scale, MCST modified card sorting test, $T M T$ trail making test, $T o L-D X$ tower of London-Drexler University, COWAT controlled word association task, RAVLT Rey auditory verbal learning task, $R T$ reaction time, $S D R T$ standard deviation of RT, No. number

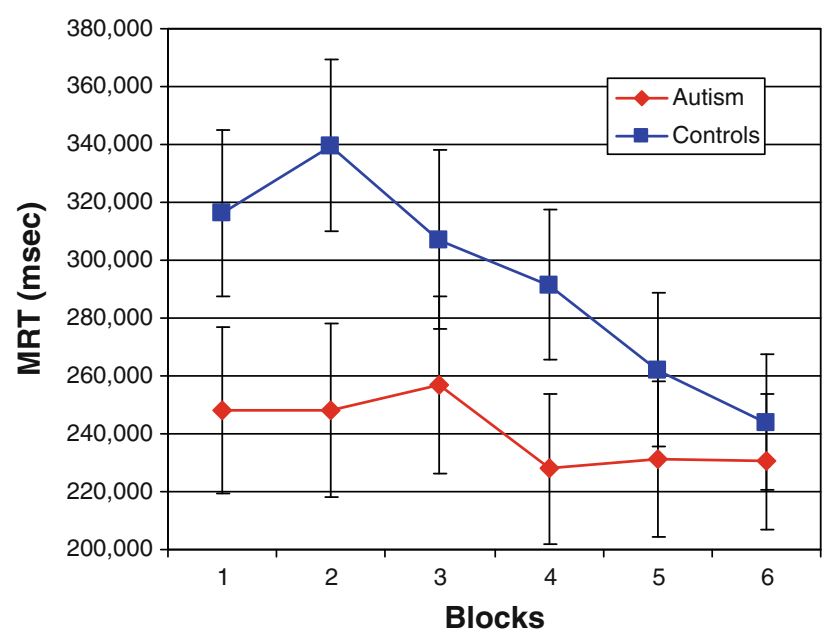

Fig. 1 Mean reaction time (MRT, with standard errors) across the six blocks for the autism group (red line) and the control group (blue line). Only in block 2 there is a significant difference between the autism group and the controls (see "Results" section for details) (Color figure online)

\section{Exploratory Regression Analyses}

Regression analyses with age, group, and interaction between age and group as predictors were conducted for each of the main dependent measures to determine whether age has a different impact for the autism and control group. For most dependent measures there was neither an effect of age nor an interaction effect of age and group. Hence, age did not explain a significant amount of variance in the tested models (for the group findings please see results of group comparisons) for processing speed, attention, working memory, cognitive flexibility, and planning. Moreover, in none of these domains age had a different effect in elderly with autism as compared to controls. The explained variance ranged from $1 \%$ (ToL-DX) till 32\% (TMT). However, some interesting patterns emerged for fluency and visual and verbal memory. ${ }^{2}$

\section{Fluency}

Thirty-three percent of the variance for verbal letter fluency was explained by the model $(p<.001)$. By inspecting the beta $(\beta)$ coefficient, it was established that there was a negative relationship between age and total number of correctly named words $(\beta=-.64 ; p<.001)$. This indicates that with increasing age, elderly gave less correct responses. Moreover, the interaction between age and

\footnotetext{
$\overline{2}$ Please note that the interactions remained significant when $\log$ transformations of the dependent variables were performed which shows that these interactions can be interpreted as valid interactions.
} 


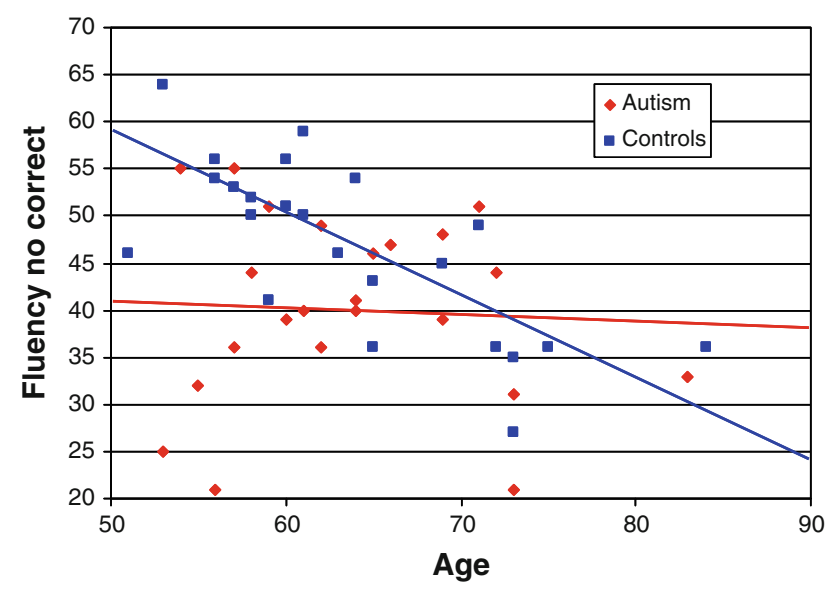

Fig. 2 Relationship between age (x-axis) and performance on the fluency task ( $y$-axis) for the autism group (red line) and the control group (blue line). This figure illustrates that age has a more pronounced effect in the control group as compared to the autism group (Color figure online)

group was significant $(\beta=2.33 ; p<.04)$, which means that age has a different effect in the autism group as compared to the controls. Visual inspection reveals that age has a more pronounced effect in the controls than in the autism group (see Fig. 2).

\section{Visual Memory}

Forty-eight percent of the variance for WMS immediate recall was explained by the model $(p<.001)$. It appears that there was a negative relationship between age and the degree of resemblance between the original and the copied figure $(\beta=-.26 ; p<.10)$, although this was not significant. The interaction between age and group was significant $(\beta=2.93 ; p<.005)$, which means that age has another effect in the autism group as compared to the controls. Visual inspection shows that age has a less pronounced effect in the controls than in the autism group (see Fig. 3). Age had a significant effect on WMS delayed recall $(\beta=-$ $.35 ; p<.05)$. Thus, with increasing age, visual memory performance decreases. However, the interaction between age and group was not significant, which suggests that age had a similar effect on both groups. The explained variance for WMS delayed recall was 35\% $(p<.001)$.

\section{Verbal Memory}

For number of correctly reproduced words at both immediate and delayed recall, the model was significant (both $p<.001$; immediate recall $34 \%$, delayed recall $31 \%$ ). However, only for immediate recall there was a negative relationship with age $(\beta=-.36 ; p<.04)$, so increasing age implies a decrease in verbal memory performance.

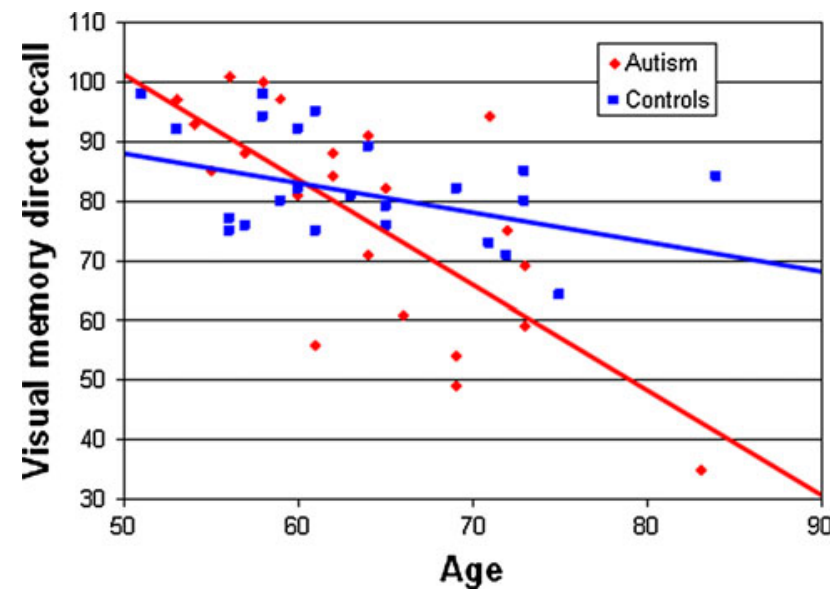

Fig. 3 Relationship between age ( $x$-axis) and performance on the visual memory task (direct recall; $y$-axis) for the autism group (red line) and the control group (blue line). This figure illustrates that age has a more pronounced effect in the autism group as compared to the control group (Color figure online)

However, there were no significant interactions between age and group.

\section{Discussion}

The major aim of this study was to determine in which cognitive domains elderly with autism have deficits. The current results suggest that elderly with HFA show impairments in sustained attention, working memory, and fluency, while other cognitive domains are intact. Different developmental patterns emerged for fluency and visual memory in elderly with HFA and controls.

In children and adults with autism deficits have been observed in planning, and cognitive flexibility (Hill 2004). In elderly with autism these deficits do not seem to be present. This might suggest that the deficits observed at young age disappear when aging, which is in line with findings from previous studies in children (Happe et al. 2006; Pellicano 2010). As age did not have a specific effect on planning in participants aged between 51 and 83 years, a speculative hypothesis is that the deficit disappears before the age of 50. A longitudinal study is needed to determine whether individuals with autism catch up with their cognitive development for some EF domains (Happe et al. 2006; Pellicano 2010). If so, this would imply that deficits present in childhood or young adulthood are transitory and will disappear when aging. This might, for example, be due to different effects of age on brain development. With respect to cortical volume it seems that the age related reduction seen in specific brain areas of healthy people is less profound in people with autism (Raznahan et al. 2010), which might result in smaller decrease in cognitive abilities 
(see also McAlonan et al. 2002). Alternatively, although speculative, it could be that individuals with HFA have already acquired compensatory strategies to cope with their cognitive challenges which they can use when they get older (i.e., safeguard hypothesis).

An alternative explanation for the aforementioned findings is that the tasks used were not sensitive enough to determine whether elderly with HFA still have problems in planning, and cognitive flexibility. With respect to planning, this alternative explanation seems implausible, as the task we used (ToL) has repeatedly been used to show that deficits are present in children and adults with autism (Joseph et al. 2005; Pellicano et al. 2006; Robinson et al. 2009; Verte et al. 2005; Wallace et al. 2009; Zinke et al. 2010). With respect to flexibility, the findings in children and adults with autism are not consistent. However, it seems that adults with autism already succeed on the cognitive flexibility tasks we administered (Barnard et al. 2008; Hill 2004; Hill and Bird 2006), as is also the case for elderly with HFA. Therefore, in future studies alternative tasks may be useful to determine whether or not the planning and cognitive flexibility deficits indeed disappear.

With respect to the other cognitive domains, the pattern of findings in elderly with HFA seems to be similar to the pattern seen in children and adults with autism. The deficits in sustained attention, working memory, and fluency (Bramham et al. 2009; Geurts et al. 2004; Johnson et al. 2007; Spek et al. 2008; Zinke et al. 2010) continue to be present, while visual and verbal memory are still intact (Bowler et al. 2009; Williams et al. 2006). Hence, it could well be that for most cognitive domains, aging in elderly with HFA runs in parallel with typical aging. Moreover, a recent study focusing on brain anatomy (cortical thickness and cortical surface area) in people with autism (aged 10 through 60 years) showed that there are no differences in the developmental pattern of the frontal cortex between people with and people without autism (Raznahan et al. 2010). Our findings discussed so far suggest that there might be different developmental patterns for different aspects of cognition (see also Luna et al. 2007), as some deficits remain stable and others seem to disappear. However, from these findings it is not clear whether some of the deficits that remain might become more severe over time.

In line with the aging literature we found that with increasing age, performance decreases for both visual memory (Vakil et al. 2010) and fluency (Clark et al. 2009). However, the effect of aging differed between individuals with and without HFA. Whereas for fluency, the performance decrease was steeper for elderly without autism, for visual memory elderly with HFA showed a steeper performance decrease with increasing age. This latter pattern might suggest an accelerated decrease (i.e., double jeopardy).
One could hypothesize that aging and autism form a double jeopardy. The main hypothesis in one of the most dominant models of cognitive aging, the scaffolding theory of aging and cognition (Cabeza 2002; Goh and Park 2009; Park and Reuter-Lorenz 2009), is that when the aging brain degenerates, it will adapt to this degeneration by using different (or additional) brain areas as a compensatory mechanism. This is thought to be a normal adaptation of the brain to reduce the impact of the age-related brain degeneration on cognitive processes. Hence, if the aging brain would not reorganize, the cognitive performance of elderly would be worse. Various neuroimaging findings suggest that similar compensatory processes are already taking place in individuals with autism in adolescence and adulthood. It has been reported that in people with autism, more (and larger) brain areas are recruited during performance on EF tasks to obtain a performance similar to controls (Koshino et al. 2005; Schmitz et al. 2006; Takarae et al. 2007; Thakkar et al. 2008; but patterns of underactivation have also been reported; Kana et al. 2007; Luna et al. 2002; Minshew et al. 2002; Schmitz et al. 2006; Solomon et al. 2009). If normal age-related compensatory processes cannot be recruited in autism because the cognitive reserves are already in use at young age, one would expect a faster decline in cognitive performance in elderly with autism than in healthy controls. However, this account does not explain why the accelerated decrease was only observed for immediate reproduction of visual information.

The present study suffers from some limitations complicating the interpretation of these findings. First, a relatively small number of participants over 60 years of age were included, while it is known that various cognitive problems in healthy aging start to emerge only at the age of 60 (Nilsson et al. 2009; Treitz et al. 2007). Visual inspection of the figures (Figs. 2, 3) suggests that especially after the age of 75 performance is affected differently. Second, even though we verified the clinical diagnosis with a self-report questionnaire, we did not use other diagnostic measures to control whether the autism diagnoses were indeed correct. Third, most of the participants with autism were high functioning and received their autism diagnosis in late adulthood, which implies that the current findings may not be representative for the whole autism population. Moreover, we used the DART as a test of IQ, but this test of verbal intelligence might overestimate the IQs of some participants with autism as individuals with autism are sometimes very good in the automatic, rigid decoding of words, without having corresponding abilities in other skills. Nonetheless, given that for most participants the educational level was at or above average, we believe that we did solely include high functioning individuals. 
In sum, aging might have a different impact in people with psychiatric disorders, like autism, than in controls, but the effect of aging in HFA depends largely on the cognitive domain under study. Although the aging trajectory for people with HFA is typical for most cognitive domains (i.e., parallel development), it is atypical and diverges from the normal pattern for some cognitive domains. On the one hand there is suggestive evidence for a double jeopardy aging pattern, while on the other hand there is evidence for a safeguard aging pattern. This divergence of developmental patterns for different cognitive domains has been observed in autism with respect to the cognitive development from childhood to adulthood as well (Luna et al. 2007). The current findings show that knowledge regarding autism based on studies in childhood and adulthood cannot be translated directly to elderly with autism, as different deficits are present depending of the age of individual with autism. Before we can draw firm conclusions, the current findings need to be replicated in a large study in which more elderly above 75 years of age should be included. Also, cognitive domains under study need to be extended to social cognition, as social impairments form an important part of the challenges that elderly with autism encounter.

Acknowledgments We want to thank all participants and the Dutch Association of Autism (NVA). We thank Bas Peters and Thomas Meindertsma for their assistance in data-collection and we thank Ben Schmand for his advice regarding the selection of tasks that are commonly used in aging studies, and we thank him for his comments on the first draft of this paper.

Open Access This article is distributed under the terms of the Creative Commons Attribution Noncommercial License which permits any noncommercial use, distribution, and reproduction in any medium, provided the original author(s) and source are credited.

\section{References}

Ambery, F. Z., Russell, A. J., Perry, K., Morris, R., \& Murphy, D. G. (2006). Neuropsychological functioning in adults with Asperger syndrome. Autism, 10, 551-564.

American Psychiatric Association. (1994). Diagnostic and statistical manual of mental disorders (4th ed.). Washington, DC: American Psychiatric Association.

American Psychiatric Association. (2000). DSM-IV-TR: Diagnostic and statistical manual of mental disorders (4, text rev. ed.). Arlington, VA: American Psychiatric Publishing.

Barnard, L., Muldoon, K., Hasan, R., O’Brien, G., \& Stewart, M. (2008). Profiling executive dysfunction in adults with autism and comorbid learning disability. Autism, 12, 125-141.

Benton, A. L., \& Hamsher, K. de. S. (1976). Multilingual aphasia examination. Iowa City: University of Iowa.

Bowler, D. M., Limoges, E., \& Mottron, L. (2009). Different verbal learning strategies in autism spectrum disorder: Evidence from the Rey auditory verbal learning test. Journal of Autism and Developmental Disorders, 39, 910-915.

Bramham, J., Ambery, F., Young, S., Morris, R., Russell, A., Xenitidis, K., et al. (2009). Executive functioning differences between adults with attention deficit hyperactivity disorder and autistic spectrum disorder in initiation, planning and strategy formation. Autism, 13, 245-264.

Cabeza, R. (2002). Hemispheric asymmetry reduction in older adults: The HAROLD model. Psychology and Aging, 17, 85-100.

Caffarra, P., Vezzadini, G., Dieci, F., Zonato, F., \& Venneri, A. (2004). Modified card sorting test: Normative data. Journal of Clinical and Experimental Neuropsychology, 26, 246-250.

Carriere, J. S., Cheyne, J. A., Solman, G. J., \& Smilek, D. (2010). Age trends for failures of sustained attention. Psychology and Aging, $25,569-574$.

Cederlund, M., Hagberg, B., Billstedt, E., Gillberg, I. C., \& Gillberg, C. (2008). Asperger syndrome and autism: A comparative longitudinal follow-up study more than 5 years after original diagnosis. Journal of Autism and Developmental Disorders, 38, $72-85$.

Clark, L. J., Gatz, M., Zheng, L., Chen, Y. L., McCleary, C., \& Mack, W. J. (2009). Longitudinal verbal fluency in normal aging, preclinical, and prevalent Alzheimer's disease. American Journal of Alzheimers Diseases and Other Dementias, 24, 461-468.

Constantino, J. N., \& Todd, R. D. (2005). Intergenerational transmission of subthreshold autistic traits in the general population. Biological Psychiatry, 57, 655-660.

Culbertson, W. C., \& Zillmer, E. A. (2001). The tower of London DX (TOLDX) manual. North Tonawanda, NY: Multi-Health Systems.

Davis, S. W., Dennis, N. A., Daselaar, S. M., Fleck, M. S., \& Cabeza, R. (2008). Que PASA? The posterior-anterior shift in aging. Cerebral Cortex, 18, 1201-1209.

De la Marche, W., Steyaert, J., Scholte, E., Dorst, M., Van Berckelaer-Onnes, I., \& Noens, I. (2009). Social Responsiveness Scale: Standardization and validation of the Dutch adult version. International Meeting for Autism Research. Chicago, May 7-9, 2009.

Friedman, D., Nessler, D., Cycowicz, Y. M., \& Horton, C. (2009). Development of and change in cognitive control: A comparison of children, young adults, and older adults. Cognitive, Affective, and Behavioral Neuroscience, 9, 91-102.

Galluzzi, S., Beltramello, A., Filippi, M., \& Frisoni, G. B. (2008). Aging. Neurological Sciences, 29(Supplement 3), 296-300.

Geurts, H. M., Corbett, B., \& Solomon, M. (2009). The paradox of cognitive flexibility in autism. Trends in Cogntivie Science, 13, $74-82$.

Geurts, H. M., Grasman, R. P., Verte, S., Oosterlaan, J., Roeyers, H., van Kammen, S. M., et al. (2008). Intra-individual variability in ADHD, autism spectrum disorders and Tourette's syndrome. Neuropsychologia, 46, 3030-3041.

Geurts, H. M., Verté, S., Oosterlaan, J., Roeyers, H., \& Sergeant, J. A. (2004). How specific are executive functioning deficits in Attention Deficit Hyperactivity Disorder and autism? Journal of Child Psychology and Psychiatry, 45, 836-854.

Gilbert, S. J., Bird, G., Brindley, R., Frith, C. D., \& Burgess, P. W. (2008). Atypical recruitment of medial prefrontal cortex in autism spectrum disorders: An fMRI study of two executive function tasks. Neuropsychologia, 46, 2281-2291.

Gilhooly, K. J., Phillips, L. H., Wynn, V., Logie, R. H., \& Della Sala, S. (1999). Planning processes and age in the five-disc tower of London task. Thinking and Reasoning, 5, 339-361.

Goh, J. O., \& Park, D. C. (2009). Neuroplasticity and cognitive aging: The scaffolding theory of aging and cognition. Restorative Neurology and Neuroscience, 27, 391-403.

Goldstein, G., Johnson, C. R., \& Minshew, N. J. (2001). Attentional processes in autism. Journal of Autism and Developmental Disorders, 31, 433-440.

Griffith, E. M., Pennington, B. F., Wehner, E. A., \& Rogers, S. J. (1999). Executive functions in young children with autism. Child Development, 70, 817-832. 
Gunning-Dixon, F. M., Brickman, A. M., Cheng, J. C., \& Alexopoulos, G. S. (2009). Aging of cerebral white matter: A review of MRI findings. International Journal of Geriatric Psychiatry, 24, 109-117.

Happe, F., Booth, R., Charlton, R., \& Hughes, C. (2006). Executive function deficits in autism spectrum disorders and attentiondeficit/hyperactivity disorder: Examining profiles across domains and ages. Brain and Cognition, 61, 25-39.

Hill, E. L. (2004). Evaluating the theory of executive dysfunction in autism. Developmental Review, 24, 189-233.

Hill, E. L., \& Bird, C. M. (2006). Executive processes in Asperger syndrome: Patterns of performance in a multiple case series. Neuropsychologia, 44, 2822-2835.

Howlin, P., Goode, S., Hutton, J., \& Rutter, M. (2004). Adult outcome for children with autism. Journal of Child Psychology and Psychiatry, 45, 212-229.

James, I. A., Mukaetova-Ladinska, E., Reichelt, F. K., Briel, R., \& Scully, A. (2006). Diagnosing Aspergers syndrome in the elderly: A series of case presentations. International Journal of Geriatric Psychiatry, 21, 951-960.

Johnson, K. A., Robertson, I. H., Kelly, S. P., Silk, T. J., Barry, E., Daibhis, A., et al. (2007). Dissociation in performance of children with ADHD and high-functioning autism on a task of sustained attention. Neuropsychologia, 45, 2234-2245.

Joseph, R. M., McGrath, L. M., \& Tager-Flusberg, H. (2005). Executive dysfunction and its relation to language ability in verbal school-age children with autism. Developmental Neuropsychology, 27, 361-378.

Just, M. A., Cherkassky, V. L., Keller, T. A., Kana, R. K., \& Minshew, N. J. (2007). Functional and anatomical cortical underconnectivity in autism: Evidence from an fMRI study of an executive function task and corpus callosum morphometry. Cerebral Cortex, 17, 951-961.

Kana, R. K., Keller, T. A., Minshew, N. J., \& Just, M. A. (2007). Inhibitory control in high-functioning autism: decreased activation and underconnectivity in inhibition networks. Biological Psychiatry, 62, 198-206.

Kanner, L. (1943). Autistic disturbances of affective contact. Nervous Child, 2, 217-250.

Keary, C. J., Minshew, N. J., Bansal, R., Goradia, D., Fedorov, S., Keshavan, M. S., et al. (2009). Corpus callosum volume and neurocognition in autism. Journal of Autism and Developmental Disorders, 39, 834-841.

Koshino, H., Carpenter, P. A., Minshew, N. J., Cherkassky, V. L., Keller, T. A., \& Just, M. A. (2005). Functional connectivity in an fMRI working memory task in high-functioning autism. Neuroimage, 24, 810-821.

Lopez, B. R., Lincoln, A. J., Ozonoff, S., \& Lai, Z. (2005). Examining the relationship between executive functions and restricted, repetitive symptoms of autistic disorder. Journal of Autism and Developmental Disorders, 35, 445-460.

Luna, B., Doll, S. K., Hegedus, S. J., Minshew, N. J., \& Sweeney, J. A. (2007). Maturation of executive function in autism. Biological Psychiatry, 61, 474-481.

Luna, B., Minshew, N. J., Garver, K. E., Lazar, N. A., Thulborn, K. R., Eddy, W. F., et al. (2002). Neocortical system abnormalities in autism: An fMRI study of spatial working memory. Neurology, 59, 834-840.

MacPherson, S. E., Phillips, L. H., \& Della Sala, S. (2002). Age, executive function, and social decision making: A dorsolateral prefrontal theory of cognitive aging. Psychology and Aging, 17, 598-609.

McAlonan, G. M., Daly, E., Kumari, V., Critchley, H. D., van Amelsvoort, T., Suckling, J., et al. (2002). Brain anatomy and sensorimotor gating in Asperger's syndrome. Brain, 125, 1594-1606.
McCurry, S. M., Gibbons, L. E., Uomoto, J. M., Thompson, M. L., Graves, A. B., Edland, S. D., et al. (2001). Neuropsychological test performance in a cognitively intact sample of older Japanese American adults. Archives of Clinical Neuropsychology, 16, 447-459.

Minshew, N. J., Meyer, J., \& Goldstein, G. (2002). Abstract reasoning in autism: A dissociation between concept formation and concept identification. Neuropsychology, 16, 327-334.

Mora, F., Segovia, G., \& del Arco, A. (2007). Aging, plasticity and environmental enrichment: Structural changes and neurotransmitter dynamics in several areas of the brain. Brain Research Reviews, 55, 78-88.

Nelson, H. (1976). A modified card sorting test sensitive to frontal lobe defects. Cortex, 12, 313-324.

Nelson, H. E. (1982). National reading test manual. London: National Hospital.

Nilsson, L. G., Sternang, O., Ronnlund, M., \& Nyberg, L. (2009). Challenging the notion of an early-onset of cognitive decline. Neurobiology of Aging, 30, 521-524. Discussion 530-523.

Ozonoff, S., \& McEvoy, R. E. (1994). A longitudinal study of executive function and theory of mind development in autism. Development and Psychopathology, 6, 415-431.

Park, D. C., \& Reuter-Lorenz, P. (2009). The adaptive brain: aging and neurocognitive scaffolding. Annual Reviews of Psychology, 60, 173-196.

Pellicano, E. (2010). The development of core cognitive skills in autism: A 3-year prospective study. Child Development, 81, $1400-1416$.

Pellicano, E., Maybery, M., Durkin, K., \& Maley, A. (2006). Multiple cognitive capabilities/deficits in children with an autism spectrum disorder: "Weak" central coherence and its relationship to theory of mind and executive control. Development and Psychopathology, 18, 77-98.

Pennington, B. F., \& Ozonoff, S. (1996). Executive functions and developmental psychopathology. Journal of Child Psychology and Psychiatry, 37, 51-87.

Price, L., Said, K., \& Haaland, K. Y. (2004). Age-associated memory impairment of logical memory and visual reproduction. Journal of Clinical Experimental Neuropsychology, 26, 531-538.

Rapin, I., \& Tuchman, R. F. (2008). Autism: Definition, neurobiology, screening, diagnosis. Pediatric Clinics of North America, $55,1129-1146$.

Raznahan, A., Toro, R., Daly, E., Robertson, D., Murphy, C., Deeley, Q., et al. (2010). Cortical anatomy in autism spectrum disorder: An in vivo MRI study on the effect of age. Cerebral Cortex, 20, $1332-1340$

Reitan, R. M., \& Wolfson, D. (1985). The Halstead-Reitan Neuropsycholgical test battery: Therapy and clinical interpretation. Tucson, AZ: Neuropsychological Press.

Rey, A. (1964). L'examen clinique en psychologie. Paris, France: Presses Universitaires de France.

Robertson, I. H., Manly, T., Andrade, J., Baddeley, B. T., \& Yiend, J. (1997). "Oops!": Performance correlates of everyday attentional failures in traumatic brain injured and normal subjects. Neuropsychologia, 35, 747-758.

Robinson, S., Goddard, L., Dritschel, B., Wisley, M., \& Howlin, P. (2009). Executive functions in children with autism spectrum disorders. Brain and Cognition, 71, 362-368.

Salthouse, T. A. (2004). What and when of cognitive aging. Current Directions in Psychological Science, 13, 140-144.

Salthouse, T. A., \& Meinz, E. J. (1995). Aging, inhibition, workingmemory, and speed. Journals of Gerontology Series B-Psychological Sciences and Social Sciences, 50, 297-306.

Salthouse, T. A., \& Miles, J. D. (2002). Aging and time-sharing aspects of executive control. Memory \& Cognition, 30, 572-582. 
Schmand, B., Geerlings, M. I., Jonker, C., \& Lindeboom, J. (1998). Reading ability as an estimator of premorbid intelligence: Does it remain stable in emergent dementia? Journal of Clinical and Experimental Neuropsychology, 20, 42-51.

Schmand, B., Lindeboom, J., \& Van Harskamp, F. (1992). Nederlandse leestest voor volwassenen. Lisse: Swets \& Zeitlinger.

Schmitz, N., Rubia, K., Daly, E., Smith, A., Williams, S., \& Murphy, D. G. (2006). Neural correlates of executive function in autistic spectrum disorders. Biological Psychiatry, 59, 7-16.

Seltzer, M. M., Shattuck, P., Abbeduto, L., \& Greenberg, J. S. (2004). Trajectory of development in adolescents and adults with autism. Mental Retardation Developmental Disability Research Reviews, 10, 234-247.

Sergeant, J. A., Geurts, H., \& Oosterlaan, J. (2002). How specific is a deficit of executive functioning for attention-deficit/hyperactivity disorder? Behavioural Brain Research, 130, 3-28.

Shafritz, K. M., Dichter, G. S., Baranek, G. T., \& Belger, A. (2008). The neural circuitry mediating shifts in behavioral response and cognitive set in autism. Biological Psychiatry, 63, 974-980.

Solomon, M., Ozonoff, S. J., Ursu, S., Ravizza, S., Cummings, N., Ly, S., et al. (2009). The neural substrates of cognitive control deficits in autism spectrum disorders. Neuropsychologia, 47, $2515-2526$.

Spek, A. A., Scholte, E. M., \& van Berckelaer-Onnes, I. A. (2008). Brief report: The use of WAIS-III in adults with HFA and Asperger syndrome. Journal of Autism and Developmental Disorders, 38, 782-787.

Sullivan, E. V., \& Pfefferbaum, A. (2006). Diffusion tensor imaging and aging. Neuroscience and Biobehavioural Reviews, 30, $749-761$

Takarae, Y., Minshew, N. J., Luna, B., \& Sweeney, J. A. (2007). Atypical involvement of frontostriatal systems during sensorimotor control in autism. Psychiatry Research, 156, 117-127.

Thakkar, K. N., Polli, F. E., Joseph, R. M., Tuch, D. S., Hadjikhani, N., Barton, J. J., et al. (2008). Response monitoring, repetitive behaviour and anterior cingulate abnormalities in autism spectrum disorders (ASD). Brain, 131, 2464-2478.

Treitz, F. H., Heyder, K., \& Daum, I. (2007). Differential course of executive control changes during normal aging. Neuropsychology, Development, and Cognition, Section B, Aging, Neuropsychology and Cognition, 14, 370-393.
Vakil, E., Greenstein, Y., \& Blachstein, H. (2010). Normative data for composite scores for children and adults derived from the Rey auditory verbal learning test. The Clinical Neuropsychologist, 24, 662-677.

Van den Burg, W., Saan, R. J., \& Deelman, B. G. (1985). 15- Woordentest. Provisional manual. Groningen: University Hospital, Department of Neuropsychology.

Verhaeghen, P., \& Cerella, J. (2002). Aging, executive control, and attention: A review of meta-analyses. Neuroscience and Biobehavioral Reviews, 26, 849-857.

Verte, S., Geurts, H. M., Roeyers, H., Oosterlaan, J., \& Sergeant, J. A. (2005). Executive functioning in children with autism and Tourette syndrome. Developmental Psychopathology, 17, $415-445$.

Volkmar, F. R., Lord, C., Bailey, A., Schultz, R. T., \& Klin, A. (2004). Autism and pervasive developmental disorders. Journal of Child Psychology and Psychiatry, 45, 135-170.

Wallace, G. L., Silvers, J. A., Martin, A., \& Kenworthy, L. E. (2009). Brief report: Further evidence for inner speech deficits in autism spectrum disorders. Journal of Autism and Developmental Disorders. doi:10.1007/s10803-009-0802-8.

Wechsler, D. (1987). Wechsler Memory Scale-Revised manual. New York: The Psychological Corporation.

Wechsler, D. (2002). Wechsler Memory Scale (3rd UK ed.). Oxford: Pearson Assessment.

Wilde, N. J., Strauss, E., \& Tulsky, D. S. (2004). Memory span on the Wechsler Scales. Journal of Clinical and Experimental Neuropsychology, 26, 539-549.

Williams, D. L., Goldstein, G., \& Minshew, N. J. (2006). Neuropsychologic functioning in children with autism: Further evidence for disordered complex information-processing. Child Neuropsychology, 12, 279-298.

Zinke, K., Fries, E., Altgassen, M., Kirschbaum, C., Dettenborn, L., \& Kliegel, M. (2010). Visuospatial short-term memory explains deficits in tower task planning in high-functioning children with autism spectrum disorder. Child Neuropsychology, 16, 229-241.

Zook, N., Welsh, M. C., \& Ewing, V. (2006). Performance of healthy, older adults on the Tower of London revised: Associations with verbal and nonverbal abilities. Neuropsychology, Development, and Cognition, Section B, Aging, Neuropsychology and Cognition, 13, 1-19. 